\title{
On some behavior spaces and Riemann-Roch theorem on open Riemann surfaces ${ }^{1)}$
}

\author{
By
}

Turgut BASKAN

(Communicated by Prof. Kusunoki, Dec. 2, 1974)

\section{Introduction.}

The purpose of this paper is to obtain a formulation of the Riemann-Roch theorem on open Riemann surfaces by using the real Hilbert space of square integrable complex differentials and introducing a special $\Lambda_{p}$-behavior space, as has been done by Shiba [9]. In our case, only $A$-periods are normalized, and $B$-periods are completely arbitrary and this character of our behavior space is in contrast with $\Lambda_{0}$-behavior in [9]. Besides this, the period normalization in this paper gives much hope to obtain some relations between these behavior spaces and the classical works. Also it seems that in a similar way, we can get the Riemann-Roch theorem by treating the complex Hilbert space. But in this case the ideal boundary becomes small [7], [8]. To get the Riemann-Roch theorem for general open Riemann surfaces, Kusunoki [2,3] made restrictions only on the real part of differentials. In Kusunoki's line, some works have been done. As in [9], our formulation of the Riemann-Roch theorem is valid for general surfaces with large boundaries.

The author wishes to express his deepest gratitude to Professor Y. Kusunoki for his valuable help and kindly encouragement. The author's thanks also go to M. Shiba and K. Matsui with whom the author had many valuable discussions.

1) This work has been done while the author was a researcher at Kyoto University under Japanese Government scholarship for 1973-75. 


\section{Preliminaries.}

The totality of square integrable complex differentials on a Riemann surface $W$ forms a Hilbert space over the complex field $\boldsymbol{C}$, if we introduce the usual inner product defined by

$$
\left(\lambda_{1}, \lambda_{2}\right)=\iint_{W} \lambda_{1} \wedge \bar{\lambda}_{2}^{*}=\iint_{W}\left(a_{1} \bar{a}_{2}+b_{1} \bar{b}_{2}\right) d x d y
$$

where $\lambda_{j}=a_{j}(z) d x+b_{j}(z) d y$ with local parameter $z=x+i y$. We denote it by $\tilde{\Lambda}=\tilde{\Lambda}(W)$. As usual $\bar{\lambda}=\bar{a} d x+\bar{b} d y$ and $\lambda^{*}=-b d x+a d y$ stand for the complex conjugate and conjgate of $\lambda$ respectively. The norm in $\tilde{\Lambda}$ is denoted by $\|\lambda\|=(\lambda, \lambda)^{1 / 2}$. Square integrable real differentials on $W$ also form a Hilbert space $\Gamma=\Gamma(W)$ over the real field $\boldsymbol{R}$ with the same inner product as above. It can be easily checked that $\tilde{\Lambda}$ forms a linear space over $\boldsymbol{R}$, and in this meaning we denote it by $\Lambda=\Lambda(W)$. $\Lambda$ forms a real Hilbert space with respect to the new inner product defined by

$$
\left\langle\lambda_{1}, \lambda_{2}\right\rangle=\operatorname{Re}\left(\lambda_{1}, \lambda_{2}\right) .
$$

The norm in $A$ will be denoted by $\| \cdot$ It is trivial that $\|=$ and so $\tilde{\Lambda}$ and $\Lambda$ have the same topological structure.

It should be noticed that, through this paper, the notations $\Gamma$ and $\Lambda$ are different from those in Ahlfors-Sario [1]. With only these exceptions we follow Ahlfors-Sario [1] for notations and terminology. For instance $\Gamma_{c}, \Gamma_{e}, \Gamma_{c o}, \Gamma_{e o}, \Gamma_{h}, \cdots$ will be used to denote the subspaces of the real Hilbert space $\Gamma$, and also $\Lambda_{e}, \Lambda_{e}, \Lambda_{c o}, \Lambda_{e o}, \Lambda_{h}, \cdots$ will stand for corresponding subspaces of $\Lambda$. The orthogonality relation between these last subspaces certainly is taken with respect to the inner product $\langle$,$\rangle . The following orthogonal decompositions$ are valid (cf. [9]):

$$
\begin{array}{lll}
\Lambda_{c}=\Gamma_{c}+i \Gamma_{c}, & \Lambda_{c o}=\Gamma_{c o}+i \Gamma_{c o}, & \Lambda_{h}=\Lambda_{c} \cap \Lambda_{c}{ }^{*} \\
\Lambda_{c}=\Lambda_{h}+\Lambda_{e o}, & \Lambda_{h}=\Lambda_{h s e}^{*}+\Lambda_{h m}, & \Lambda=\Lambda_{h} \dot{+} \Lambda_{e o} \dot{+} \Lambda_{e o}^{*} .
\end{array}
$$

The following lemma is frequently used in the sequel.

Lemma 1. 1. Let $\Omega$ be a canonical regular region on $W$, and $\boldsymbol{\Xi}(W)=\left\{A_{j}, B_{j}\right\}^{g}{ }_{j=1}$ a canonical homology basis on $W$ modulo di- 
viding cycles, such that $\Xi \cap \bar{\Omega}$ forms a canonical homology basis on $\bar{\Omega}$ modulo $\partial \Omega$. If $\varphi_{1}, \varphi_{2}$ are $C^{1}$-differentials which are semiexact and closed respectively, then

$$
\left(\varphi_{1}, \varphi_{2}^{*}\right)_{\Omega}=\int_{\partial \Omega}\left(\int \varphi_{1}\right) \bar{\varphi}_{2}+\sum_{\Omega}\left(\int_{A_{j}} \varphi_{1} \int_{B_{j}} \bar{\varphi}_{2}-\int_{B_{j}} \varphi_{1} \int_{A_{j}} \bar{\varphi}_{2}\right) .
$$

This can be proved by cutting $\Omega$ along $A_{j}, B_{j}$, and applying Green's formula.

Note that because of closedness of $\varphi_{2}$ the integral $\int_{\partial \Omega}\left(\int \varphi_{1}\right) \bar{\varphi}_{2}$ is independent of the additive constant of $\int \varphi_{1}$.

\section{2. $A_{p}$-behavior space.}

Definition 2. 1. A linear subspace $\Lambda_{p}$ of $\Lambda_{h s e}$ will be called a behavior space if

(1) There exists a closed subspace $\Lambda_{1}$ of $\Lambda_{h s e}$ such that

$$
\Lambda_{p} \supset \Lambda_{1}+i \Lambda_{1}^{\perp *}
$$

where $\Lambda_{1}{ }^{\perp}$ is the orthogonal complement of $\Lambda_{1}$ in $\Lambda_{h}$

(2) $\left\langle\lambda_{p}, i \lambda_{p}{ }^{*}\right\rangle=0$ for each $\lambda_{p} \in \Lambda_{p}$

(3) $\quad \int_{A_{j}} \lambda_{p}=0, j=1,2, \cdots$ for every $\lambda_{p} \in \Lambda_{p}$.

From this definition it is easy to verify that if $\Lambda_{p}$ is a behavior space, so is $\bar{\Lambda}_{p}$, where $\bar{\Lambda}_{p}=\left\{\bar{\lambda}_{p}: \lambda_{p} \in \Lambda_{p}\right\}$.

Definition 2.2. A meromophic differential defined on a neighborhood $U$ of the ideal boundary $\beta$ of $W$ is said to have $\Lambda_{p}$-behavior if there exist $\lambda_{p} \in \Lambda_{p}, \lambda_{e 0} \in \Lambda_{e o} \cap \Lambda^{1}$ such that on a neighborhood $U$ of $\beta$

$$
\varphi=\lambda_{p}+\lambda_{e o}
$$

Definition 2.3. A meromorphic function $f$ (not necessarily single-valued) defined near $\beta$ is said to have $\Lambda_{p}$-behavior if differential $d f$ has $\Lambda_{p}$-behavior in the above sense.

\section{The existence and uniqueness theorems.}

Theorem 3. 1. (uniqueness). Let $\varphi$ be a first kind differential 
which has $\Lambda_{p}$-behavior. Then it is identically zero if

$$
\int_{A_{j}} \varphi=0 \quad(j=1,2, \cdots, g)
$$

where $g(\leq \infty)$ is the genus of $W$.

Proof. It should be observed that the condition in the theorem is only for finite number of $A_{j}$. Since $\varphi$ has $\Lambda_{p}$-behavior, there exist $\lambda_{p} \in \Lambda_{p}$, $\lambda_{e o} \in \Lambda_{e o} \cap \Lambda^{1}$ such that on a neighborhood $U$ of $\beta \varphi$ can be written as

$$
\varphi=\lambda_{p}+\lambda_{e o} .
$$

Now let $\Omega$ be a canonical regular region on $W$ such that its relative boundary $\partial \Omega$ is contained in $U$. We may assume that $\Xi \cap \bar{\Omega}$ forms a canonical homology basis of $\bar{\Omega}$ modulo the border. Then, by Lemma 1.1 and $\int_{\boldsymbol{A}} \varphi=0(j=1,2, \cdots$,$) we can write$

$$
\begin{aligned}
\|\varphi\|_{\Omega}{ }^{2} & =\|\varphi\|_{\Omega}^{2}=(\varphi, \varphi)_{\Omega}=-i\left(\varphi, \varphi^{*}\right)_{e} \\
& =i \int_{\partial \Omega}\left(\int \varphi\right) \bar{\varphi}-i \sum_{\Omega}\left(\int_{A_{j}} \varphi \int_{B_{j}} \bar{\varphi}-\int_{B_{j}} \varphi \int_{A_{j}} \bar{\varphi}\right) \\
& =i \int_{\partial \Omega}\left(\int \varphi\right) \bar{\varphi}=i \int_{\partial \Omega}\left(\int\left(\lambda_{p}+\lambda_{e o}\right)\right)\left(\overline{\lambda_{p}+\lambda_{e o}}\right) \\
& =-i\left(\lambda_{p}+\lambda_{e o}, \lambda_{p}{ }^{*}+\lambda_{e o}^{*}\right)_{\Omega}+i \sum\left(\int_{A_{j}} \lambda_{p} \int_{B_{g}} \bar{\lambda}_{p}-\int_{B_{j}} \lambda_{p} \int_{A_{j}} \bar{\lambda}_{p}\right) .
\end{aligned}
$$

From the condition (3) in the definition of $\Lambda_{p}$-behavior we obtain

$$
\|\varphi\|_{\Omega}^{2}=\|\varphi\|_{\Omega}^{2}=\left(\lambda_{p}, i \lambda_{p}{ }^{*}\right)_{\Omega}-i \varepsilon_{\Omega}
$$

where $\varepsilon_{\Omega}=\left(\lambda_{e o}, \lambda_{p}{ }^{*}\right)_{\Omega}+\left(\lambda_{p}, \lambda_{e o}^{*}\right)_{\Omega}+\left(\lambda_{e o}, \lambda_{e o}^{*}\right)_{\Omega}$. By making use of the orthogonal decompositions in section 1 , it follows that $\lim _{\Omega \rightarrow W} \varepsilon_{\Omega}=0$ Then, we get the equality

$$
\|\varphi\|^{2}=\left(\lambda_{p}, i \lambda_{p}^{*}\right)=\left\langle\lambda_{p}, i \lambda_{p}^{*}\right\rangle .
$$

The right side is also zero because of the condition (2) in Definition 2. 1 , and so we get $\varphi \equiv 0$.

Now we will prove the existence of certain first kind differentials which have $\Lambda_{p}$-behavior. 
Theorem 3.2. Let $\alpha_{j} \neq 0$ be given complex numbers. Then there exist square integrable first kind differentials $\phi_{\alpha_{j}}\left(B_{j}\right)$ which have the following properties:

(i) $\phi_{\alpha,}\left(B_{j}\right)$ have $\Lambda_{p}$-behavior

(ii) $\quad \int_{\boldsymbol{A}_{k}} \phi_{\alpha_{j}}\left(B_{j}\right)= \begin{cases}-\alpha_{j} & (k=j) \\ 0 & (k \neq j)\end{cases}$

(iii) The $\phi_{\alpha_{j}}\left(B_{j}\right)$ are uniquely determined for each $j$.

Proof. The cycles $B_{j}$ can be regarded as oriented analytic Jordan curves. Let $R$ be a relatively compact ring domain containing a $B$, and $v$ be a $C^{2}$-function on $R-B$, defined as follows:

$$
v=\left\{\begin{array}{l}
\alpha_{j} \text { on the left side of } B_{j} \\
0 \text { on the right side of } B_{j} .
\end{array}\right.
$$

Then $v$ can be extended to $W-B$, such that it becomes a $C^{2}$-function with relatively compact support in $W$. Denote the extension by $\widehat{v}$. Then $d \widehat{v} \in \Lambda_{c}{ }^{1}(W)$ and so it can bewritten as:

$$
d \widehat{v}=\lambda_{1}+\lambda_{1}{ }^{\perp}+\lambda_{e o}
$$

where

$$
\lambda_{1} \in \Lambda_{1}, \quad \lambda_{1}{ }^{\perp} \in \Lambda_{1}{ }^{\perp}, \quad \Lambda_{1}+i \Lambda_{1}^{*}{ }^{\perp} \subset \Lambda_{p} .
$$

Now we set

$$
\phi_{\alpha j}\left(B_{j}\right)=\lambda_{1}{ }^{\perp}+i\left(\lambda_{1}{ }^{\perp}\right)^{*}=d \widehat{v}-\left(\lambda_{1}-i \lambda_{1}^{* \perp}\right)-\lambda_{e o}=d \widehat{v}-\lambda_{p}-\lambda_{e o} .
$$

It can be seen from this equation that $\phi_{\alpha_{j}}\left(B_{j}\right)$ is a first kind differential and has $\Lambda_{p}$-behavior, since $d \widehat{v}$ has compact support. Also for any cycle $\gamma$

$$
\int_{r} \phi_{\alpha_{j}}\left(B_{j}\right)=\alpha_{j}\left(B_{j} \times \gamma\right)-\int_{r} \lambda_{p}
$$

Now if we take $A_{k}$ instead of $\gamma$, then (ii) is satisfied. The uniqueness follows easily from Theorem 3.1.

To prove the existence of second and third kind differentials we need the following lemma [10]. 
Lemma 3. 1. Let $\Omega$ be a regularly imbedded connected subregion of $W$ rohose relative boundary $\partial \Omega$ is compact, and $V$ be the complement of $\bar{\Omega}$. For any closed $C^{1}$-differential $\sigma$ defined on a neighborhood of $\bar{V}$, the following two statements are equivalent:

(i) $\sigma \mid V$, the restriction of $\sigma$ onto $V$, can be extended as a closed $C^{1}$-differential $\hat{\sigma}$ on $W$ such that the support of $\hat{\sigma}$ has a compact intersection with $\bar{Q}$.

(ii) $\int_{\partial \Omega} \sigma=0$.

Theorem 3. 3. Let $\theta_{j}$ be an analytic singularity given at each point $p_{j}$ on $W(j=1,2, \cdots, n)$. Consider a differential $\theta$ which is equal to $\theta$, near $p_{j}$ and the sum of residues of $\theta$ is zero. Then there exists a differential $\varphi=\varphi_{\theta}$ such that

(i ) $\varphi$ has $A_{p}$-behavior

(ii) $\varphi$ is regular analytic except at $p_{j}(j=1,2, \cdots, n)$

(iii) $\varphi$ has singularity $\theta$, that is, $\|\theta-\varphi\|_{\sigma_{j}}<\infty$ for a punctured neighborhood $U_{j}$ of $p_{j}(j=1,2, \cdots, n)$.

The proof can be carried out in the same manner as AhlforsSario [1], Shiba [9], if we use our orthogonal decomposition

$$
\Lambda=\Lambda_{1}+\Lambda_{1}{ }^{\perp}+\Lambda_{e o}+\Lambda_{e o}^{*} \text {. }
$$

Namely define

$$
\tau=\hat{\theta}-\lambda_{1}-\lambda_{e o}^{\prime}=\lambda_{1}{ }^{\perp}+\lambda_{e o}^{\prime \prime *}+i \hat{\theta}^{*} .
$$

Then $\tau$ is a complex harmonic differential with singularity $\theta$. Consequently $\lambda_{e o}^{\prime}, \lambda_{e o}^{\prime \prime} \in \Lambda_{e o} \cap \Lambda^{1}$, since $\tau \in \Lambda^{1}, \hat{\theta} \in C^{1}, \lambda_{1} \in \Lambda_{1}$. If we set $\varphi=\frac{1}{2}\left(\tau+i \tau^{*}\right)$ it is easily seen that $\varphi$ has the desired properties.

Remark. We can see that the differentials constructed above are uniquely determined, if we require that $\varphi$ should satisfy

$$
\int_{A_{j}} \varphi=0 \quad(j=1,2, \cdots, g) .
$$

Now we show that this normalization is always possible.

Indeed, let $x_{j}$ be $A_{j}$-periods of $\varphi$ such that only a finite number 
of $x_{j}$ are different from zero. We set

$$
\varphi_{p}=\varphi+\sum_{j} \phi_{x_{j}}\left(B_{j}\right)
$$

It is clear that $\varphi_{p}$ preserves the singularity, and satisfies the normalization:

$$
\int_{A_{j}} \varphi_{p}=\int_{A_{j}} \varphi+\sum_{j} \int_{A_{j}} \phi_{x_{j}}\left(B_{j}\right)=\int_{A_{j}} \varphi-x_{j}=0
$$

As for uniqueness we need only Theorem 3.1.

The following normalized differentials whose existence is guaranteed by Theorem 3.3, and the holomorphic (first kind) differentials $\phi_{\alpha_{j}}\left(B_{j}\right)$ obtained by Theorem 3.2 , will play an important role in the proof of the Riemann-Roch theorem.

(I) $\varphi_{p_{j}, n}$, (resp. $\widetilde{\varphi}_{p_{j}, n}$ ): differential with $\Lambda_{p}$-behavior, regular analytic except at $p_{j}$ where it has singularity $d z / z_{j}{ }^{n}$ (resp. $\left.i d z / z_{j}{ }^{n}\right) \quad(n=2,3, \cdots)$

(II) $\phi_{p_{j}, q_{j}}$, (resp. $\widetilde{\psi}_{p_{j}, q_{j}}$ ): meromorphic differential with $\Lambda_{p}$-behavior, which has residues 1 at $p_{j},-1$ at $q_{j}$ (resp. $i$ at $p_{j},-i$ at $q_{j}$ ) and regular elsewhere.

\section{Dual boundary behaviors.}

Definition 4. 1. Let $\Lambda_{p}{ }^{(k)}=\Lambda_{p}\left(\Lambda_{1}{ }^{(k)}, 0, C\right) \quad(k=1,2)$ be two behavior spaces corresponding to the subspaces $\Lambda_{1}{ }^{(1)}, \Lambda_{1}{ }^{(2)} \subset \Lambda_{h s e}$. We say that $\Lambda_{p}{ }^{(1)}$-behavior and $\Lambda_{p}{ }^{(2)}$ behavior are dual to each other if for all $\lambda_{p}{ }^{(1)} \in \Lambda_{p}{ }^{(1)}, \lambda_{p}{ }^{(2)} \in \Lambda_{p}{ }^{(2)}$

$$
\left(\lambda_{p}{ }^{(1)}, \overline{\lambda_{p}{ }^{(2) *}}\right)=0 \quad\left(\Leftrightarrow\left\langle\lambda_{p}{ }^{(1)}, \ddot{\lambda}_{p}{ }^{(2)} *\right\rangle=\left\langle\lambda_{p}{ }^{(1)}, i{\overline{\lambda_{p}}}^{(2) *}\right\rangle=0\right) .
$$

The following lemma is a nice consequence of this definition.

Lemma 4. 1. Suppose that $\Lambda_{p}=\Lambda_{p}\left(\Lambda_{1}, 0, C\right)$ is a behavior which satisfies the condition:

(i) $\quad\left(\lambda_{p}, i \lambda_{p}{ }^{1 *}\right)=0 \quad\left(\Leftrightarrow\left\langle\lambda_{p}, i \lambda_{p}{ }^{1 *}\right\rangle=\left\langle\lambda_{p}, \lambda_{p}{ }^{1 *}\right\rangle=0\right)$

for all $\lambda_{p}, \lambda_{p}{ }^{1} \in \Lambda_{p}$. 
Then $\Lambda_{p}$-behavior and $\overline{\Lambda_{p}}$-behavior are dual to each other.

Proof. Since $\bar{\Lambda}_{p}$ is a behavior space, we need only check the condition in definition 4.1. For this purpose take $\lambda_{p}, \lambda_{p}{ }^{1} \in \Lambda_{p}$ then by (i) we get

$$
\begin{aligned}
\left(\lambda_{p},\left(\overline{\left.\overline{\lambda_{p}}\right)^{*}}\right)\right. & =\left\langle\lambda_{p}, \lambda_{p}{ }^{1 *}\right\rangle+i\left\langle\lambda_{p}, i \lambda_{p}{ }^{1 *}\right\rangle \\
& =0
\end{aligned}
$$

The following lemma $[2,3]$, will be used in the proof of Riemann-Roch theorem. Therefore we prove it in our terminology.

Lemma 4.2. Let $\Lambda_{p}{ }^{(1)}$ and $\Lambda_{p}{ }^{(2)}$ be dual boundary behaviors to each other. Let $\varphi$ be an abelian differential (of first or second kind) with $\Lambda_{p}{ }^{(1)}$-behavior and $\phi$ any abelian differential with $\Lambda_{p}{ }^{(2)}$ behavior. Let $W_{0}$ be the planar surface obtained from $W$ by cutting along $A_{j}$ and $B_{j}$ cycles. Then,

(i) there exists a single valued meromorphic function $f$ on $W_{0}$ such that $d f=\varphi$,

(ii) $2 \pi i \sum \operatorname{Res} f \psi=-\sum_{j=1}^{g}\left(\int_{A_{j}} \varphi \int_{B_{j}} \psi-\int_{B_{j}} \varphi \int_{A_{j}} \psi\right)$.

Proof. (i) is obvious by assumptions. To prove (ii) we apply Lemma 1.1 to the region $\Omega_{0}$ obtained from a sufficiciently large canonical region $\Omega$ by taking off mutually disjoint parametric disks about the singularities of $\varphi$ and $\phi$. We may suppose that $\Xi \cap \bar{\Omega}$ forms a canonical homology basis of $\bar{\Omega}$ modulo $\partial \Omega$ then

$$
2 \pi i \sum \operatorname{Res} f \psi=-\sum_{\Omega}\left(\int_{A_{j}} \varphi \int_{B_{j}} \psi-\int_{B_{j}} \varphi \int_{A_{j}} \psi\right)+\int_{\partial \Omega} f \psi
$$

By assumption we know that $\varphi=\lambda_{p}{ }^{(1)}+\lambda_{e o}, \psi=\lambda_{p}{ }^{(2)}+\lambda_{e o}^{1}$ near the ideal boundary, in particular near $\partial \Omega$. By use of Lemma 1.1, and from the definitions of $\Lambda_{p}$ and its dual behavior we can write

$$
\begin{aligned}
\int_{\partial \Omega} f \psi & =-\left(\lambda_{p}{ }^{(1)}, \overline{\lambda_{p}{ }^{(2) *}}\right)_{\Omega}+\sum_{\Omega}\left(\int_{A_{j}} \lambda_{p}{ }^{(1)} \int_{B_{j}} \lambda_{p}{ }^{(2)}-\int_{B_{j}} \lambda_{p}{ }^{(1)} \int_{A_{j}} \lambda_{p}{ }^{(2)}\right)+\varepsilon_{\Omega} \\
& =-\left(\lambda_{p}{ }^{(1)}, \overline{\left.\lambda_{p}{ }^{(2) *}\right)_{\Omega}+\varepsilon_{\Omega} \rightarrow 0 \quad(\Omega \rightarrow W) .}\right.
\end{aligned}
$$


Thus we get the desired result.

\section{The Riemann-Roch Theorem.}

Let $\delta=\delta_{p} / \delta_{q}$ be a finite divisor on $W$, where $\delta_{p}=P_{1}^{{ }^{m}} \cdot P_{2}{ }^{m_{2}} \ldots P_{r}{ }^{m_{r}}$ and $\delta_{q}=q_{1}{ }^{n_{1}} q_{2}{ }^{n_{2}} \cdots q_{s}{ }^{n_{s}}$ are disjoint integral divisors. Let $\Lambda_{p}{ }^{(1)}$ and $\Lambda_{p}{ }^{(2)}$ be dual boundary behaviors. We consider the following sets which evidently form linear spaces over $\boldsymbol{R}$ :

$S\left(\Lambda_{p}{ }^{(1)} ; 1 / \delta\right)=\{f:$ (i) single valued meromorphic function on $W$, (ii) has $\Lambda_{p}^{(1)}$-behavior, (iii) is multiple of of $\left.1 / \hat{0}\right\}$

$M\left(\Lambda_{p}{ }^{(1)} ; 1 / \delta_{p}\right)=\{f:$ (i) is a multi-valued meromorphic function on $W$. (ii) has $\Lambda_{p}{ }^{(1)}$-behavior, (iii) is a multiple of $1 / \delta_{p}$ (iv) periods of $d f$ are normalized, i.e., $\left.\int_{A} d f=0\right\}$

$D\left(\Lambda_{p}{ }^{(2)} ; \delta\right)=\{\alpha$ : (i) a meromorphic differential on $W$, (ii) has $\Lambda_{p}{ }^{(2)}$-behavior, (iii) is a multiple of $\left.\delta\right\}$

$E\left(\Lambda_{p}{ }^{(2)} ; 1 / \delta_{q}\right)=\{\alpha$ : (i) a meromorphic differential on $W$, (ii) has $\Lambda_{p}{ }^{(2)}$-behavior, (iii) is a multiple of $\left.1 / \delta_{q}\right\}$

In the case that $\delta_{q} \neq 1$ we identify the elements $f_{1}, f_{2}$ of $M$ if and only if $f_{1}-f_{2}=$ constant.

The following well-known algebraic lemma should be provided.

Lemma 5.1. Let $X$ and $Y$ be two linear spaces over a field $K$, and consider a biliner form $(x, y)$ defined over $X \times Y$. Denote the left kernel by $X_{0}$ and the right kernel by $Y_{0}$. If the quotient space $X / X_{0}$ is finite dimensional, then there is an isomorphism $X / X_{0} \simeq Y / Y_{0}$.

Theorem 5. 1. (Riemann-Roch). Suppose that $\Lambda_{p}{ }^{(1)}$-and $\Lambda_{p}{ }^{\left({ }^{2}\right)}$ behaviors are dual to each other. Let $\delta=\delta_{p} / \delta_{q}$ be a finite divisor on $W$, where $\delta_{p}$ and $\delta_{q}$ are disjoint integral divisors. Then

$$
\begin{gathered}
\operatorname{dim} S\left(\Lambda_{p}^{(1)} ; 1 / \hat{\delta}\right)=2\left[\operatorname{deg} \delta_{p}+1-\min \left(\operatorname{deg} \delta_{q}, 1\right)\right] \\
-\left[\operatorname{dim} E\left(\Lambda_{p}{ }^{(2)} ; 1 / \delta_{q}\right) / D\left(\Lambda_{p}{ }^{(2)} ; \hat{\jmath}\right)\right] .
\end{gathered}
$$

Proof. We follow essentially the proof of Kusunoki [2]. We define a function $h_{p}(f, \alpha)$ on $M \times E$ by

$$
h_{p}(f, \alpha)=\operatorname{Re}\left(\sum_{j}^{1} \operatorname{Res}_{p_{j}} f \alpha\right) \quad \text { for } f \in M, \alpha \in E .
$$


Since $\alpha$ is regular at each $p_{j}$, additive constants of $f$ have no effect on the residues of $f \alpha$ at each $p_{j}$. Therefore $h_{p}(f, \alpha)$ is well-defined. Then by Lemma 4.2 we can write

$$
h_{p}(f, \alpha)=\frac{1}{2 \pi} \operatorname{Im}\left[\sum_{j=1}^{g} \int_{B_{j}} d f \int_{A_{j}} \alpha\right]-\operatorname{Re}\left[\sum_{k} \underset{q_{k}}{\operatorname{Res} .} f \alpha\right]
$$

since $d f$ is normalized, i.e., $\int_{A_{j}} d f=0$. Thus, if $f$ belongs to the leftkernel of $h_{p}(f, \alpha)$, i.e., $0=h_{p}(f, \alpha)$ for every $\alpha \in E$, then we get $\operatorname{Im} \int_{B_{k}} d f=0, \operatorname{Re} \int_{B_{k}} d f=0$ by taking $\alpha \equiv \phi_{1}\left(B_{k}\right)$ and $\alpha \equiv \phi_{i}\left(B_{k}\right)$ respectively. Thus $\int_{B_{k}} d f=0$. Therefore $f$ is single-valued on the whole $W$, since by assumption we already know that $\int_{A_{k}} d f=0$. If $\delta$ is an integral divisor, then $\delta=\delta_{p}$ and so $f \in S$. If $\delta$ is non-integral, then we take $\alpha \equiv \psi_{q_{1}, q_{k}}^{(2)}$. It can be seen that $\operatorname{Im} f\left(q_{1}\right)=\operatorname{Im} f\left(q_{k}\right)$ and $\operatorname{Re} f\left(q_{1}\right)$ $=\operatorname{Re} f\left(q_{k}\right) \quad(k=1,2, \cdots, s)$. Thus $f-f\left(q_{1}\right)$ has zeros at $q_{k}(2 \leq k \leq s)$. Moreover, if we take $\varphi_{q_{k}, \nu}^{(2)}$ and $\widetilde{\varphi}_{q_{k, \nu}}^{(2)}$ as $\alpha\left(1 \leq k \leq s, 2 \leq \nu \leq n_{k}\right)$ it follows that $f-f\left(q_{1}\right)$ has at least $n_{k}$ zeros at $q_{k}$. By the equivalent relation in $M$ we get $f \in S$. Conversely, it is obvious that the left-kernel of $h_{p}$ contains $S$. In a similar way we can see that $D$ is the right-kernel of $h_{p}$. Indeed, since $f \alpha$ is regular analytic at each $p_{j}$ for $f \in M$, $\alpha \in D$, then $D$ is contained in the right-kernel. The converse is proved by taking the integrals $\int \varphi_{p_{, \mu}}^{(1)}$ and $\int \widetilde{\varphi}_{p_{j, \mu}}^{(1)}$ as $f\left(1 \leq j \leq r, 2 \leq \mu \leq m_{j}+1\right)$. To get the final result we must see that $M$ is a finite-dimensional space. For $\delta_{q} \neq 1$ the following integrals span $M$;

$$
\int \varphi_{p_{\mu, \mu}^{(1)}} \text { and } \quad \int \widetilde{\varphi}_{p_{j, \mu}^{(1)}} \quad \begin{array}{ll} 
& 1 \leq j \leq r \\
& 2 \leq \mu \leq m_{j}+1
\end{array}
$$

If $\delta_{q}=1$, the above integrals and $1, i$ make a basis of $M$. So we find that

$$
\operatorname{dim} M=\left\{\begin{array}{l}
2 \sum_{j=1}^{r} m_{j}+2=2 \operatorname{deg} \hat{o}_{p}+2 \quad\left(\hat{o}_{q}=1\right) \\
2 \sum_{j=1}^{r} m_{j}=2 \operatorname{deg} \hat{o}_{p} \quad\left(\hat{o}_{q} \neq 1\right)
\end{array}\right.
$$

So in any case we have $\operatorname{dim} M=2\left[\operatorname{deg} \hat{o}_{p}+1-\min \left(\operatorname{deg} \hat{o}_{q}, 1\right)\right]$. Then we can apply Lemma 5.1 . q.e.d.

If the genus of $W$ is finite, Theorem 5.1 reduces to the follow- 
ing rather classical form:

Corollary 5.1. If $\Lambda_{p}{ }^{(1)}$-and $\Lambda_{p}{ }^{(2)}$-behaviors are dual to each other, then for any finite divisor $\delta$ on $W$

$$
\operatorname{dim} S-\operatorname{dim} D=2(\operatorname{deg} \delta-g+1) .
$$

Proof. We can find a basis for $E$ :

(a) if $\delta_{q}=1 \quad\left\{\phi_{a_{j}}^{(2)}\left(B_{j}\right), \phi_{i b_{j}}^{(2)}\left(B_{j}\right)\right\}_{j=1}^{o} \quad$ span $E$, where $a_{j}, b_{j} \in \boldsymbol{R}$.

(b) if $\delta_{q} \neq 1 \quad\left\{\phi_{a_{j}}^{(2)}\left(B_{j}\right), \phi_{i b_{j}}^{(2)}\left(B_{j}\right), \varphi_{q_{k}, \nu}^{(2)}, \widetilde{\varphi}_{q_{k}, \nu}^{(2)}, \psi_{q_{1}, q_{l}}^{(2)}, \widetilde{\psi}_{q_{1}, q_{l}}^{(2)}\right\}_{2 \leq l \leq j, 2 \leq \nu \leq n_{k}}^{1 \leq j \leq g_{2}, 1 \leq k \leq s}$

span $E$ provided that in both cases we choose $a_{j}$ and $b_{j}$ as in Theorem 2.1, then

$$
\operatorname{dim} E=\left\{\begin{array}{l}
2 g \quad\left(\delta_{q}=1\right) \\
2\left[g+\sum_{k=1}^{s}\left(n_{k}-1\right)+s-1\right] \quad\left(\delta_{q} \neq 1\right) .
\end{array}\right.
$$

So, $\operatorname{dim} E=2\left[g-\min \left(\operatorname{deg} \delta_{q}, 1\right)+\operatorname{deg} \delta_{q}\right]$ and the result easily follows from Theorem 5. 1 .

\section{Generalization.}

Divide the set of positive integers $\{1,2, \cdots, g\}$ into two disjoint sets $J_{1}, J_{2}$, and let $\left\{L_{j}\right\}$ be a set of straight lines $L_{j}\left(j \in J_{1}\right)$ passing through the origin $z=0$.

Definition 6. 1. A linear subspace $\Lambda_{p}=\Lambda_{p}\left(J_{1}, J_{2}\right)$ of $\Lambda_{h s t}$ is called a behavior space if

(1) there exists a closed subspace $\Lambda_{1}$ of $\Lambda_{h s}$ such that

$$
\Lambda_{p} \supset \Lambda_{1}+i \Lambda_{1}{ }^{1 *}
$$

where $\Lambda_{1}^{\perp}$ is the orthogonal complement of $\Lambda_{1}$ in $\Lambda_{h}$

(2) $\left\langle\lambda_{p}, i \lambda_{p}^{*}\right\rangle=0$ for each $\lambda_{p} \in \Lambda_{p}$

(3) $\int_{\boldsymbol{A}_{j},}, \lambda_{p} \in L_{j}$ if $j \in J_{1}$, and $\int_{A}, \lambda_{p}=0$ if $j \in J_{2}$.

We can similarly formulate the Riemann-Roch theorem in terms of such behavior spaces. As a special case where $J_{2}=\phi$, we have Shiba's result [9], and our result is the case $J_{1}=\phi$. Given $L_{j}$ we can prove that a behavior space $\Lambda_{p}$ actually exists. 


\section{HACETTEPE UUIVERSity, ANKARA, TURKEY.}

\section{References}

[1] Ahlfors, L. V. \& Sario, L. Rimann surfaces, Princeton Univ. Press 1960.

[2] Kusunoki, Y. Contributions to Riemann-Roch theorem, Memoirs Col. Sci. Univ. Kyoto Ser. A. Math., 31 (1958), 161-180.

[3] _ . The theory of Abelian integrals and its applications to conformal mappings, Ibid., 32 (1959), 235-258.

[4] Supplements and corrections to my former papers, Ibid. 33 (1961), 429-433.

[5] Characterizations of canonical differentials, J. Math. Kyoto Univ. 5 (1966), 197-208.

[6] Matsui, K. Convergence theorems of Abelian differentials with applications to conformal mappings, J. Math. Kyoto Univ. 15 (1975), 73-100.

[7] Rodin, B. Reproducing kernels and principal functions, Proc. Amer. Math. Soc., 13 (1962), 982-992.

[ 8 ] Royden, H. L. The Riemann-Roch theorem, Comm. Math. Helv. 34 (1960), 3751.

[9] Shiba, M. On the Riemann-Roch theorem on open Riemann surfaces, J. Math. Kyoto Univ. 11 (1971), 495-525.

[10] Yoshida, M. The method of orthogonal decompositions for differentials on open Riemann surfaces, J. Sci. Hiroshima Univ. Ser. A-1, 32 (1968), 181-210. 